\title{
Immunostimulatory AdCD40L gene therapy combined with low-dose cyclophosphamide in metastatic melanoma patients
}

\author{
Angelica Loskog ${ }^{1,6}$, Aglaia Maleka ${ }^{1,2,6}$, Sara Mangsbo ${ }^{1}$, Emma Svensson ${ }^{1}$, Christina Lundberg ${ }^{3}$, \\ Anders Nilsson ${ }^{3}$, Johan Krause ${ }^{3}$, Margrét Agnarsdóttir ${ }^{1,4}$, Anders Sundin ${ }^{3,5}$, Håkan Ahlström ${ }^{3,5}$, \\ Thomas H Tötterman ${ }^{1,7,8}$ and Gustav Ullenhag ${ }^{\star 1,2,7}$ \\ ${ }^{1}$ Department of Immunology, Genetics and Pathology, Science for Life Laboratory, Uppsala University, Dag Hammarskjoldsvag 20, \\ 75185 Uppsala, Sweden; ${ }^{2}$ Department of Oncology, Uppsala University Hospital, 75185 Uppsala, Sweden; ${ }^{3}$ Division of Radiology, \\ Uppsala University Hospital, 75185 Uppsala, Sweden; ${ }^{4}$ Department of Pathology and Cytology, Uppsala University Hospital, 75185 \\ Uppsala, Sweden and ${ }^{5}$ Department of Surgical Sciences, Uppsala University, 75185 Uppsala, Sweden
}

Background: Current approaches for treating metastatic malignant melanoma (MM) are not effective enough and are associated with serious adverse events. Due to its immunogenicity, melanoma is an attractive target for immunostimulating therapy. In this phase I/Ila study, local AdCD40L immunostimulatory gene therapy was evaluated in patients with MM.

Methods: AdCD40L is an adenovirus carrying the gene for CD40 ligand. Patients that failed standard treatments were enrolled. Six patients received four weekly intratumoral AdCD40L injections. Next, nine patients received low-dose cyclophosphamide conditioning before the first and fourth AdCD40L injection. The blood samples were collected at multiple time points for chemistry, haematology and immunology evaluations. Radiology was performed at enrolment and repeated twice after the treatment.

Results: AdCD40L was safe with mild transient reactions. No objective responses were recorded by MRI, however, local and distant responses were seen on FDG-PET. The overall survival at 6 months was significantly better when cyclophosphamide was added to AdCD40L. The patients with the best survival developed the highest levels of activated T cells and experienced a pronounced decrease of intratumoral IL8.

Conclusions: AdCD40L therapy for MM was well tolerated. Local and distant responses along with better survival in the low-dose cyclophosphamide group are encouraging.

The rising incidence and mortality rate of malignant melanoma (MM) makes it one of the most common causes of cancer-related deaths among young adults. The treatment with BRAF inhibitors, the anti-CTLA4 antibody ipilimumab and PD1/PDL1 inhibitors significantly prolongs life in those who are in a good general condition. However, prognosis remains poor. Furthermore, these new treatments are associated with serious adverse events (Weber, 2007; Sosman et al, 2012; Sullivan et al, 2013). Considering the immunogenicity of MM, other immunotherapeutic options may be of interest such as local immunostimulatory gene therapy.

AdCD40L is a replication-deficient adenovirus carrying the gene for CD40 ligand (CD40L). CD40L binds to CD40 present on

\footnotetext{
*Correspondence: Dr GJ Ullenhag; E-mail: gustav.ullenhag@igp.uu.se

${ }^{6}$ Shared first position.

${ }^{7}$ Shared senior position.

${ }^{8}$ Study sponsor.
}

Received 8 September 2015; revised 13 January 2016; accepted 1 February 2016; published online 31 March 2016

(c) 2016 Cancer Research UK. All rights reserved 0007-0920/16 
various cell types including dendritic cells (DCs) and macrophages. On CD40L/CD40 interaction, DCs mature and stimulate Th1-type immune responses (Loskog and Totterman, 2007). AdCD40L has previously been used in a clinical trial to treat patients with locally advanced urinary bladder cancer (Malmstrom et al, 2010), as well as in dogs with metastatic MM with promising effects (Westberg et al, 2013). To enhance the efficacy of immunotherapy, low-dose cyclophosphamide is widely used owing to its capacity to reduce the number of T-regulatory cells (Tregs) and to stimulate NK cells (Muranski et al, 2006). Cyclophosphamide is a chemotherapeutic that belongs to the group of alkylating agents. It has proven efficacy against various malignancies but not against MM.

We conducted an open-label phase I/IIa trial with intratumour AdCD40L injections \pm low-dose cyclophosphamide conditioning. Our aim was to assess the feasibility and effectiveness of AdCD40L treatment in patients with metastatic MM and to evaluate whether cyclophosphamide can improve the efficacy. The patients were monitored for toxicity and immune modulation as well as for tumour response.

\section{MATERIALS AND METHODS}

Trial design. The study protocol (NCT01455259) was in accordance with the International Conference of Harmonization-Good Clinical Practice guideline (ICH-GCP), the principles of the Declaration of Helsinki and regulatory guidelines. The protocol was approved by the Regional Ethical Committee and the National Medical Products Agency. The primary objective was to evaluate the feasibility of repeated cycles of intratumoral AdCD40L injections \pm cyclophosphamide conditioning with clinical and immunological responses as secondary end points. The trial comprised two parts. In the first part, (group A), six patients received four weekly ultrasound-guided intratumoral injections of $2.5 \times 10^{11}$ virus particles (VP) AdCD40L. In the second part, (group B), nine patients were conditioned with low-dose intravenous cyclophosphamide therapy $\left(300 \mathrm{mg} \mathrm{m}^{-2}\right) 1-2$ days before the first and fourth treatment with AdCD40L. Only six patients were treated with AdCD40L alone, as none of them experienced an objective response (OR). The size of the cyclophosphamide cohort was defined by applying the methodology of Gehan. The risk of terminating the study after none of the nine patients achieved an OR, despite that the treatment in reality confers up to $30 \%$ OR, is less than $5 \%$ (Gehan, 1961). The patients were monitored for a period of 10 weeks in which they were sampled for blood chemistry, haematology and immunology evaluation at multiple time points. All the patients underwent pretreatment whole-body MRI and positron emission tomography (PET), integrated with computed tomography (PET/CT), using $\left[{ }^{18}\right.$ F]fluoro-deoxy-glucose (FDG), on a Discovery ST16 PET/CT scanner (GE Healthcare, Little Chalfont, Great Britain). MRI and FDG-PET/CT were repeated 2 and 6 weeks after the last AdCD40L injection. Morphological (MRI) tumour responses were assessed according to the RECIST 1.1 criteria and tumour metabolic activity (FDG-PET/CT) by measuring the maximum standardised uptake value SUVmax at 1 hour after tracer injection. (Hwang et al, 2014). Metabolic tumour response in the injected metastasis was defined according to EORTC criteria as $\geqslant 15 \%$ decrease and metabolic progression as $\geqslant 25 \%$ increase of the SUVmax (Young et al, 1999).

AdCD40L. AdCD40L is an adenoviral serotype 5, replication deficient vector (He et al, 1998). It carries the transgene for human CD40L driven by an RSV promoter. The vector was developed and GMP produced (batch VEC407.1) at Baylor College of Medicine, Houston, TX, USA. On intratumoral injection, the virus was thawed and diluted in a final dilution of $2.5 \times 10^{11} \mathrm{VP}$ in $500 \mu$ l Ringer lactate solution. The virus was kept at $+4{ }^{\circ} \mathrm{C}$ until injection.
Analyses of biopsies and plasma. Antibodies against adenovirus were measured by the Adenovirus IgG ELISA (GenWay, San Diego, CA, USA). Biopsy lysates were generated from frozen tumour biopsies by the disruption of individual biopsies and mixing with a standard RIPA lysing buffer. Lysates and plasma samples were analysed using ProSeek Multiplex Inflammation ${ }^{96 \times 96}$ (Olink Biosciences AB, Uppsala, Sweden). The total protein level was used to normalise the samples for a possible uneven tumour cell content. Immunohistochemistry using aCD40 (clone HB14, BioLegend, San Diego, CA, USA) and aCD3 was performed by the routine diagnostics facility at the Department of Pathology and Cytology, Uppsala University Hospital.

Flow cytometry. Peripheral blood mononuclear cells (PBMCs) were analysed by flow cytometry. The cells were thawed, washed with phosphate-buffered saline (PBS) and incubated at room temperature for $10 \mathrm{~min}$ with $\mathrm{FcR}$ blocking reagent (Miltenyi Biotech, Bergisch Gladbach, Germany). The cells were incubated with antibodies targeting $\mathrm{CD} 3$ (clone:SK7), CD4 (clone:OKT4) and CD127 (clone:A019D5) for $30 \mathrm{~min}$ at $4{ }^{\circ} \mathrm{C}$ followed by fixation and permeabilisation. The cells were then incubated with an antibody targeting FoxP3 (clone:206D) for $30 \mathrm{~min}$ at $4{ }^{\circ} \mathrm{C}$. After a final wash with PBS with $0.5 \%$ bovine serum albumin (BSA), the cells were resuspended in PBS-BSA and analysed by flow cytometry.

Statistical evaluations. All the statistical analyses were made with Prism Software (Graphpad Software Inc., La Jolla, CA, USA. The difference in 6-month survival between the groups was studied using log-rank test. All the correlation analyses were performed using the Spearman's correlation test. The levels of caspase 8, CD40, IP10, CXCL9, TGF $\beta$ and IL8 pre- and post treatment were investigated using the Student's $t$-test with Welsh (unpaired) or Wilcoxon (paired) correction. A $P<0.05$ was considered significant.

\section{RESULTS}

Patients' characteristics. Fifteen patients (seven men, eight women) with metastatic MM with at least two radiologically measurable tumour lesions were enrolled. Informed consent was obtained from all the patients before enrolment. The average age of the patients was 61 years ranging from 23 to 79 years. All the patients were in good performance status (WHO performance status classification, WHO 0-1) at the time of enrolment (Table 1). They were refractory to standard treatments including surgery, chemotherapy, immunotherapy (interferon, ipilimumab), BRAF inhibitor and radiotherapy. Eight patients had ocular melanoma, five patients skin melanoma, one patient mucosal melanoma of the vulva and one patient had melanoma with a lymph node as the primary site.

Safety of AdCD40L treatments. From 2011 to 2013, 15 patients with stage IV melanoma were enrolled. There were six patients in group A receiving four weekly treatments of AdCD40L and nine patients in group $\mathrm{B}$ receiving cyclophosphamide conditioning before the first and fourth AdCD40L treatment (Figure 1A). All the four weekly injections were delivered in the same tumour lesion to measure the local and systemic effects. Because of rapid progressive disease (PD), patient \#11 received neither the fourth AdCD40L treatment nor the final cyclophosphamide conditioning.

The treatment with AdCD40L with or without cyclophosphamide was, in general, well tolerated. In particular, four patients in group A and eight in group B experienced transient grade I-II adverse events according to common terminology criteria for adverse events (CTCAE) version 4.0, such as fever, flu-like symptoms and pain at the injection site. None of the patients experienced grade IV adverse events, (Table 2). Only one patient 
Table 1. Background information and overall survival for malignant melanoma patients with advanced disease treated with AdCD40L with or without low-dose cyclophosphamide

\begin{tabular}{|c|c|c|c|c|c|c|c|c|c|}
\hline Patient number & Sex & $\begin{array}{l}\text { WHO } \\
\text { pre }\end{array}$ & $\begin{array}{l}\text { WHO } \\
\text { post }\end{array}$ & $\mathrm{Age}^{\mathrm{a}}$ & $\begin{array}{l}\text { Primary tumour } \\
\text { localisation }\end{array}$ & Treated metastasis & Ipi & CD3 infiltration ${ }^{b}$ & $\begin{array}{l}\text { Overall } \\
\text { survival }^{\mathrm{c}}\end{array}$ \\
\hline \multicolumn{10}{|c|}{ Without cyclophosphamide } \\
\hline 1 & $\mathrm{~F}$ & 0 & 1 & 72 & Ocular & Liver & No & Decreased & 7 \\
\hline 2 & $\mathrm{~F}$ & 1 & 1 & 61 & Node & $\mathrm{SC}$ & No & Increased & 11 \\
\hline 3 & M & 1 & 2 & 79 & Ocular & Liver & No & Stable & 13 \\
\hline 4 & $\mathrm{~F}$ & 0 & 0 & 68 & Ocular & Liver & No & - & 143 \\
\hline 5 & M & 0 & 0 & 77 & Skin & $\mathrm{SC}$ & No & Stable (NT) & 23 \\
\hline 6 & M & 0 & 1 & 63 & Skin & SC & No & Increased & 22 \\
\hline \multicolumn{10}{|c|}{ With cyclophosphamide } \\
\hline 7 & $\mathrm{M}$ & 0 & 0 & 23 & Ocular & Liver & No & Increased & 43 \\
\hline 8 & $\mathrm{~F}$ & 0 & 0 & 67 & Vulva & Node & No & Increased & $160+$ \\
\hline 9 & $\mathrm{~F}$ & 0 & 1 & 52 & Ocular & Parotid & No & Stable & 32 \\
\hline 10 & $\mathrm{~F}$ & 0 & 0 & 62 & Ocular & SC & No & Stable & 34 \\
\hline 11 & $\mathrm{~F}$ & $1 \mathrm{~A}$ & 3 & 45 & Skin & Node & $Y_{e s}{ }^{d}$ & $-(P)$ & 6 \\
\hline 12 & $M$ & $1 \mathrm{~A}$ & $1 \mathrm{~A}$ & 64 & Skin & Liver & Yes $^{\mathbf{e}}$ & $-(P)$ & 39 \\
\hline 13 & $\mathrm{~F}$ & 0 & 0 & 70 & Ocular & Liver & No & Increased & 28 \\
\hline 14 & $M$ & $1 \mathrm{~A}$ & $1 \mathrm{~A}$ & 51 & Skin & Node & Yes $^{f}$ & Increased & 68 \\
\hline 15 & $M$ & 1 & $1 \mathrm{~A}$ & 61 & Ocular & Liver & No & Stable & 14 \\
\hline \multicolumn{10}{|c|}{ 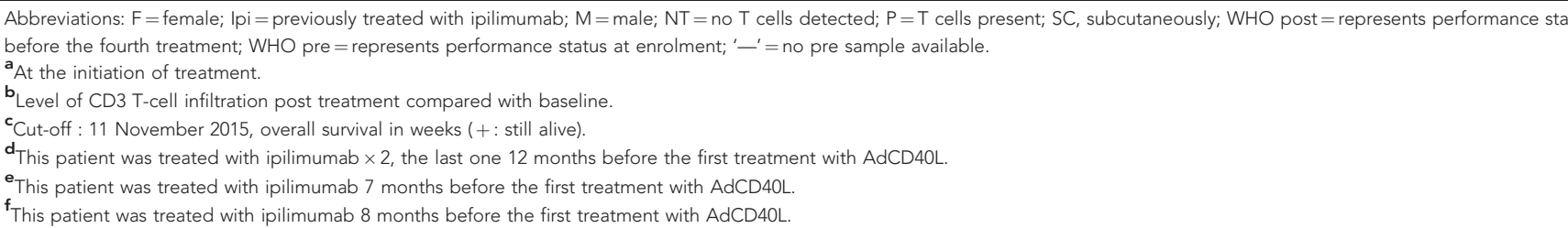 } \\
\hline
\end{tabular}

(\#12) experienced grade III symptoms; pain related to the biopsy and anaemia. However, the latter could represent anaemia of chronic disease. Moreover, a serious adverse reaction (SAE) was observed that led to the patient's hospitalisation. This male patient developed fever and shivering at the day of the third injection while also receiving a blood transfusion due to anaemia. This patient also developed round white vitiligo-like skin lesions that were observed 3 weeks after the last injection. Vitiligo, flu-like symptoms, fever and fatigue were regarded to be directly connected to the virus, whereas pain at the injection site, nausea and vomiting were considered to be due to the injection procedure.

Clinical results according to $\mathrm{PET} / \mathrm{CT}$ and whole-body MRI scan. The results of whole-body MRI (WB-MRI) are presented in Table 3. SUVmax changes of the injected metastases in FDG-PET/ $\mathrm{CT}$ are presented in Figure $2 \mathrm{~B}$ and $\mathrm{C}$. We registered two patients in group A who showed SUVmax decrease in the injected metastases. Patient \#2 had reduced tumour activity in the injected metastasis in both the post-treatment PET/CT examinations and, moreover, two distant metastases showed a SUVmax decline at the second posttreatment PET/CT. Patient \#3 had reduced tumour activity at the first post-treatment PET/CT in the injected metastasis as well as in the majority of the distant metastases. The second post-treatment PET/CT was not performed owing to deterioration of the patient's general condition. According to the first post-treatment WB-MRI, patient \#3 showed morphologically stable disease (SD), whereas patient \#2 experienced PD. Patient \#4 in group A had similar tumour activity in all the metastases in both the post-treatment PET/CT examinations compared with the baseline study. At week 9 , the patient still had SD, which continued with durable SD for 143 weeks. Four patients in group B experienced reduced metabolic activity in the injected metastasis, two of which showed reduced SUVmax in all metastases, as exemplified by the patients \#7 and \#8 (Figure 1D and C, respectively). Three patients had the same metabolic activity in all the metastases as before treatment and one had an increase in tumour activity. Patient \#11 did not undergo any post-treatment scans owing to rapid general deterioration caused by disease progression. However, according to the first post-treatment WB-MRI, only four patients were assessed as having morphologically SD and the remaining as having PD. At week 9, three patients were still experiencing SD. One of these patients, \#8, is still alive (160 weeks). At the time of study entry and before, the condition of patient \#8 was considered as palliative with lung metastases. However, reevaluation of previous examinations after the study treatment did not confirm lung involvement. Patient \#8 was, therefore, subjected to evacuation of metastatic lymph nodes in both groins (Figure 1C). The relapse was observed 2 years and 4 months after the last injection with AdCD40L.

Because of the clear metabolic improvement at PET/CT after treatment of patient \#7 and the obvious clinical improvement of patient \#14, they received an additional cycle of four AdCD40L injections combined with cyclophosphamide starting at 14 and 32 weeks, respectively, after the first treatment cycle. According to WB-MRI, retreatment was followed by morphologically PD in patient \#7. In addition, PET/CT showed increased metabolic activity in the majority of metastases although some of them showed decreased uptake. The patient died 43 weeks post enrolment. For patient \#14, WB-MRI 2 weeks after the new cycle showed morphologically $\mathrm{SD}$ while $\mathrm{PET} / \mathrm{CT}$ revealed increased metabolic activity in several metastases. This patient experienced an improved performance status from $1 \mathrm{~A}$ to 0 one month after the last AdCD40L injection. After an additional 2-week period, both WB-MRI and PET/CT showed morphologically SD and the performance status was still 0 .

Overall survival of AdCD40L-treated patients. The median survival in group A was 18 weeks and in group B 34 weeks. At 6 months, only one of six (17\%) patients was still alive in group A while seven of nine $(78 \%)$ were still alive in group B. The difference between the groups was significant according to log-rank test $(P=0.0313)$. However, the overall survival (OS) was similar in both groups $(P=0.3589$; Figure $2 \mathrm{~A})$. There was no significant difference in OS for the patients with ocular melanoma (three out of six in group A and five out of nine in group B) compared with the rest. 
Anti-adenoviral antibodies increases post treatment. Most humans have been subjected to adenoviral infections and have antibodies against the virus. All the patients enrolled in the trial had detectable levels of IgG antibodies before treatment and the levels were increased post treatment (Figure 2D). The antibody levels before or after the treatment did not correlate to survival in our study cohort and there was no clear difference in antibody levels with or without cyclophosphamide conditioning. However, patients receiving the AdCD40L injection into a liver metastasis
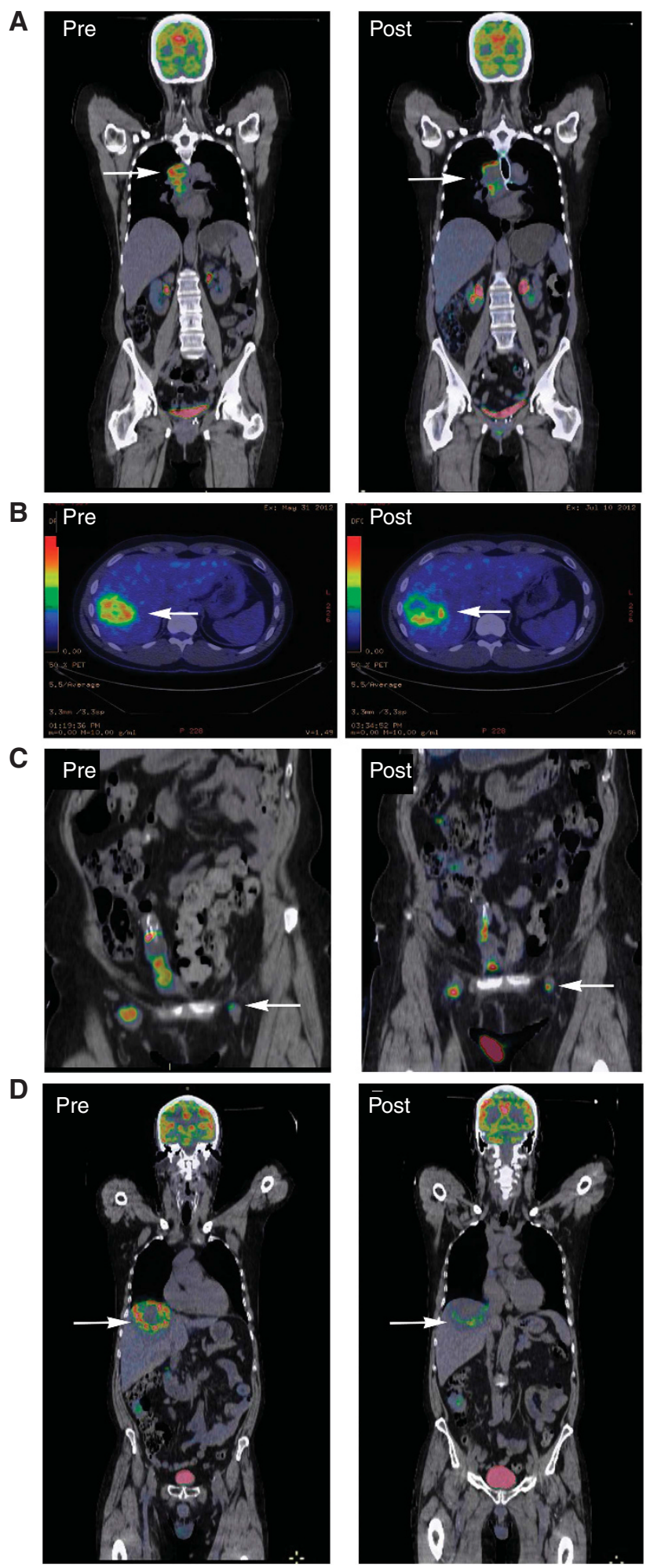

had higher antibody levels post treatment than those treated in a metastasis at a different site $(P=0.0020)$.

Cyclophosphamide did not significantly affect Treg or effector T-cell levels. The patients were evaluated for immune responses. The level of Tregs among CD4 T cells was determined by flow cytometry. In Figure 3A, the Treg level over time is demonstrated. In group $\mathrm{A}$, the Treg level was stable in the patients even if the individual levels varied. There were no changes pre and post

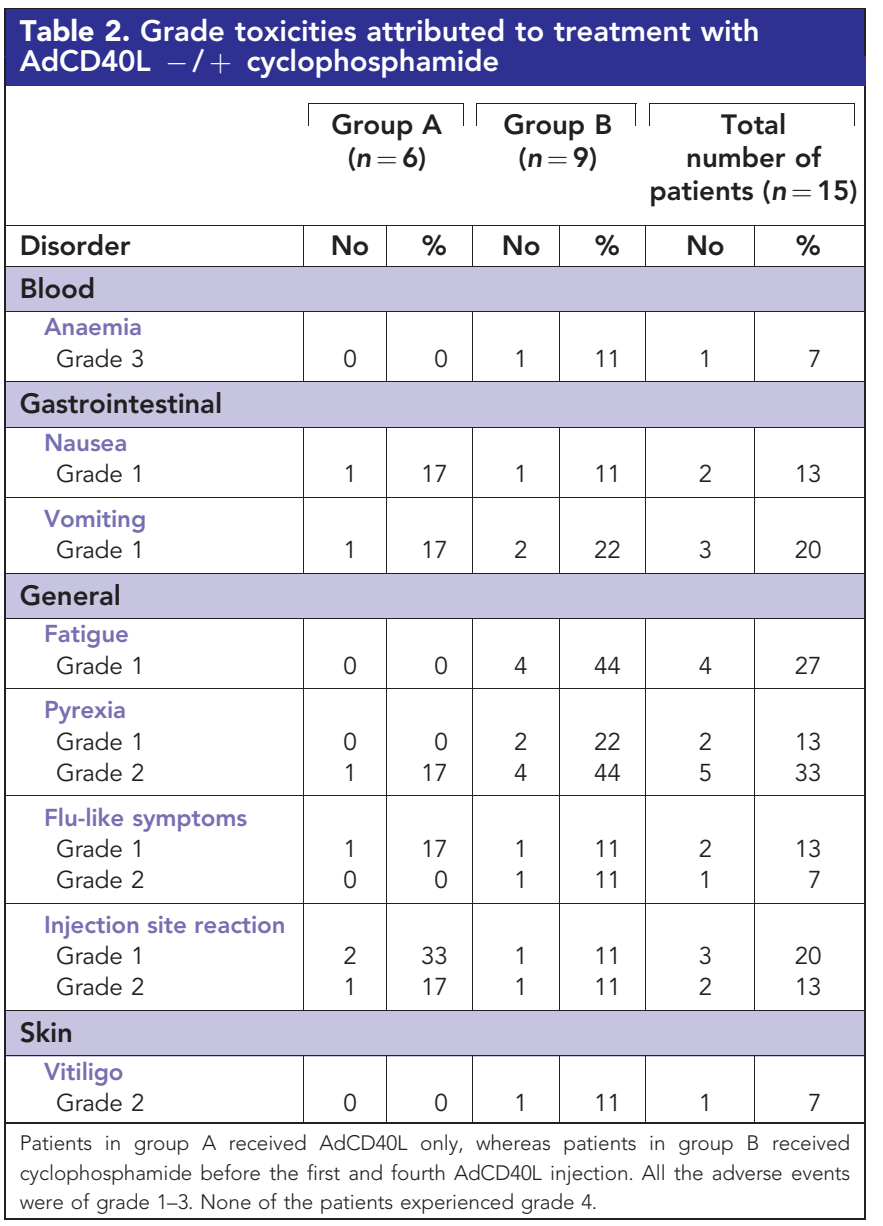

Figure 1. PET/CT fusion images showing metabolic tumour response to the treatment. (A) Patient \#2, group A, received AdCD40L injections only and showed decreased FDG uptake (SUVmax) in the mediastinal metastasis (arrow) from baseline PET/CT (Pre) to follow up PET/CT (Post) at 5 weeks after the treatment started. (B) Patient \#7, group B, showed decreased FDG uptake (SUVmax) in all metastases after four injections with AdCD4OL and low dose cyclophosphamide. PET/CT at 5 weeks (Post) shows decreased FDG uptake in a large liver metastasis compared with baseline PET/CT (Pre). (C) Patient \#8, group B, received four injections with $\mathrm{AdCD} 40 \mathrm{~L}$ and conditioning low dose

cyclophosphamide. The patient had only two metastases, in the right and left pelvis, respectively. The FDG uptake in both metastases decreased significantly from baseline PET/CT (Pre) and follow-up PET/ $\mathrm{CT}$ at 5 weeks (Post). The pelvic metastases are seen adjacent to the external iliac vessels and in the groins (arrows). This patient, after treatment, was accepted for surgical resection and is the only one who is still alive two and a half years after the last injection. (D) Patient \#12, group B, received four injections with AdCD40L and low dose cyclophosphamide and a liver metastasis (arrows) showed decreased FDG uptake 5 weeks after the last injection (PET/CT Post) as compared with baseline (Pre). 
cyclophosphamide treatment except in patient \#14 for whom a significant decrease was seen. This patient also responded well to therapy. Interestingly, patient \#7 had initially decreasing levels but

Table 3. Radiological evaluation by MRI in malignant melanoma patients with advanced disease treated with AdCD40L with or without low-dose cyclophosphamide

\begin{tabular}{|c|c|c|}
\hline \multicolumn{3}{|c|}{ MRI evaluation (RECIST 1.1) } \\
\hline Patient no & Week 5 & Week 9 \\
\hline \multicolumn{3}{|c|}{ Without cyclophosphamide } \\
\hline 1 & PD & ND \\
\hline 2 & PD & ND \\
\hline 3 & SD & PD \\
\hline 4 & SD & SD \\
\hline 5 & SD & SD \\
\hline 6 & PD & ND \\
\hline \multicolumn{3}{|c|}{ With cyclophosphamide } \\
\hline 7 & SD & SD \\
\hline 8 & SD & SD \\
\hline 9 & PD & ND \\
\hline 10 & SD & SD \\
\hline 11 & ND & ND \\
\hline 12 & PD & PD \\
\hline 13 & PD & PD \\
\hline 14 & SD & PD \\
\hline 15 & PD & PD \\
\hline \multicolumn{3}{|c|}{$\begin{array}{l}\text { Abbreviations: } \mathrm{ND}=\text { not done; } \mathrm{PD}=\text { progressive disease; } \mathrm{SD}=\text { stable disease; } \mathrm{WBMRI}= \\
\text { whole-body magnetic resonance imaging. Treatment response assessments by RECIST } 1.1 \\
\text { week: week post treatment initiation. }\end{array}$} \\
\hline
\end{tabular}

during the follow-up evaluation, the Tregs increased and this correlated to progression of his cancer. Likewise, the activated CD4 T-cell levels (CD127 + FoxP3 - of total CD3 + CD4 +) were stable in group A (Figure $3 \mathrm{~B}$ ). However, in group B, the levels were higher and the three patients that likely benefitted most from the therapy as measured by the longest OS (patient \#7, \#8 and \#14) exhibited the highest levels. Patient \#14 experienced a decrease of these cells post follow-up. The activated CD8 T cells (CD127 + of total $\mathrm{CD} 3+\mathrm{CD} 8+$ ) also remained stable during therapy and were not affected by conditioning (Figure 3C). The longest surviving patients (patient \#7, \#8 and \#14) had higher levels of activated CD8 $\mathrm{T}$ cells than the other patients with the exception of patient \#13. Patient \#15 with short overall survival (14 weeks) had a significant reduction of activated CD8 $\mathrm{T}$ cells over time. The biopsies from the patients' tumours were evaluated to determine the level of infiltrating $\mathrm{CD} 3+\mathrm{T}$ cells (Table 1 ). In group $\mathrm{A}$, two out of six patients showed increased levels. This did not include patient \#4 who lived longest in that group. Group B had in general higher numbers of infiltrating $T$ cells and four of seven evaluable patients $(\# 7, \# 8, \# 13, \# 14)$ had an increase of $\mathrm{T}$ cells post treatment, which included the three with longest survival.

CD40 levels correlate to caspase 8. Tumour lysates and plasma were evaluated for a variety of proteins. Interestingly, caspase 8 was increased in biopsies post treatment indicating cell death (Figure 4A). However, a higher presence of caspase 8 did not correlate to OS. Cell death induced by AdCD40L can be due to tumour killing not only by the cells of the immune system but also by the direct interaction with $\mathrm{CD} 40+$ tumour cells. CD 40 was
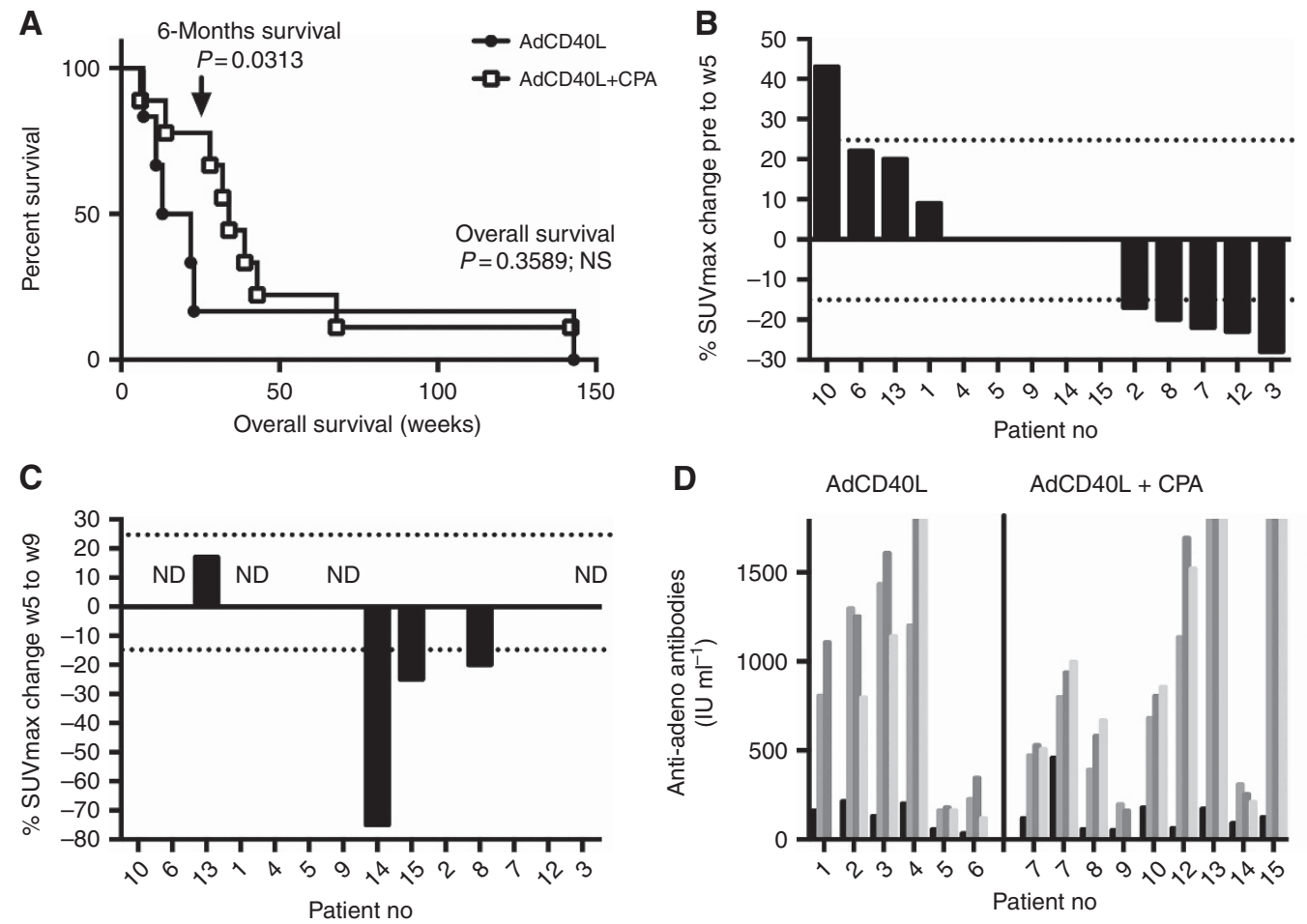

D AdCD40L AdCD40L + CPA

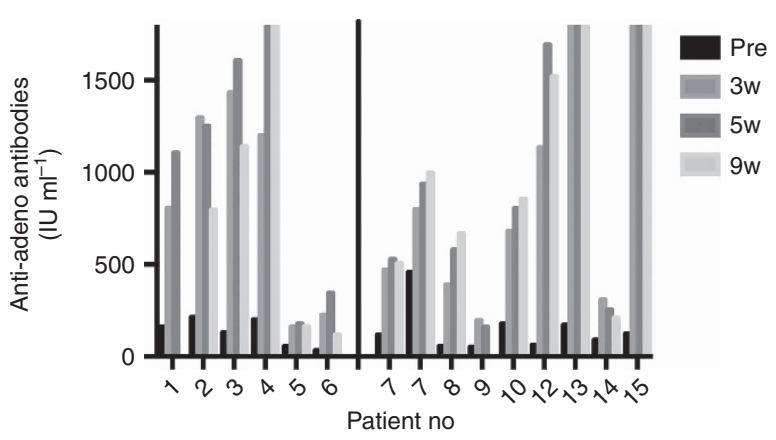

Figure 2. Survival, PET responses in injected metastases and anti-adenovirus antibodies. (A) A Kaplan-Meier survival curve for the patients treated with AdCD40L (closed circles) and AdCD40L combined with cyclophosphamide (CPA, open boxes). The patient \#11 did not complete the treatment, biopsies were not taken and she did not undergo follow-up PET/CT. Therefore the patient is excluded from figures $\mathbf{B}, \mathbf{C}$ and $\mathbf{D}$. (B) Waterfall plot of the change in SUVmax of the injected metastases from baseline PET/CT in relation to follow-up PET/CT at week 5. Black bars indicate increased uptake, grey bars metabolic regression and the flat line stable SUVmax. Dashed line at 25\% indicates the limit for PD and dashed line at $-15 \%$ indicates the limit for metabolic response according to EORTC criteria. (C) Waterfall plot showing metabolic responses (SUV max) of the injected metastases in PET scans at week 9 in relation to week 5. Patients \#14 and \#15 were metabolically stable between baseline PET and PETw5 but SUVmax decreased between PETw5 and PETw9, showing a late treatment effect. A continuous metabolic response was noted for patient \#8. (D) The levels of anti-adenovirus lgG antibodies in sera as measured with ELISA are demonstrated. The levels pre and post therapy were correlated to overall survival using Spearman correlation test and no significant differences were demonstrated. Two staples are demonstrated regarding patient \#7 because the patient was retreated. 

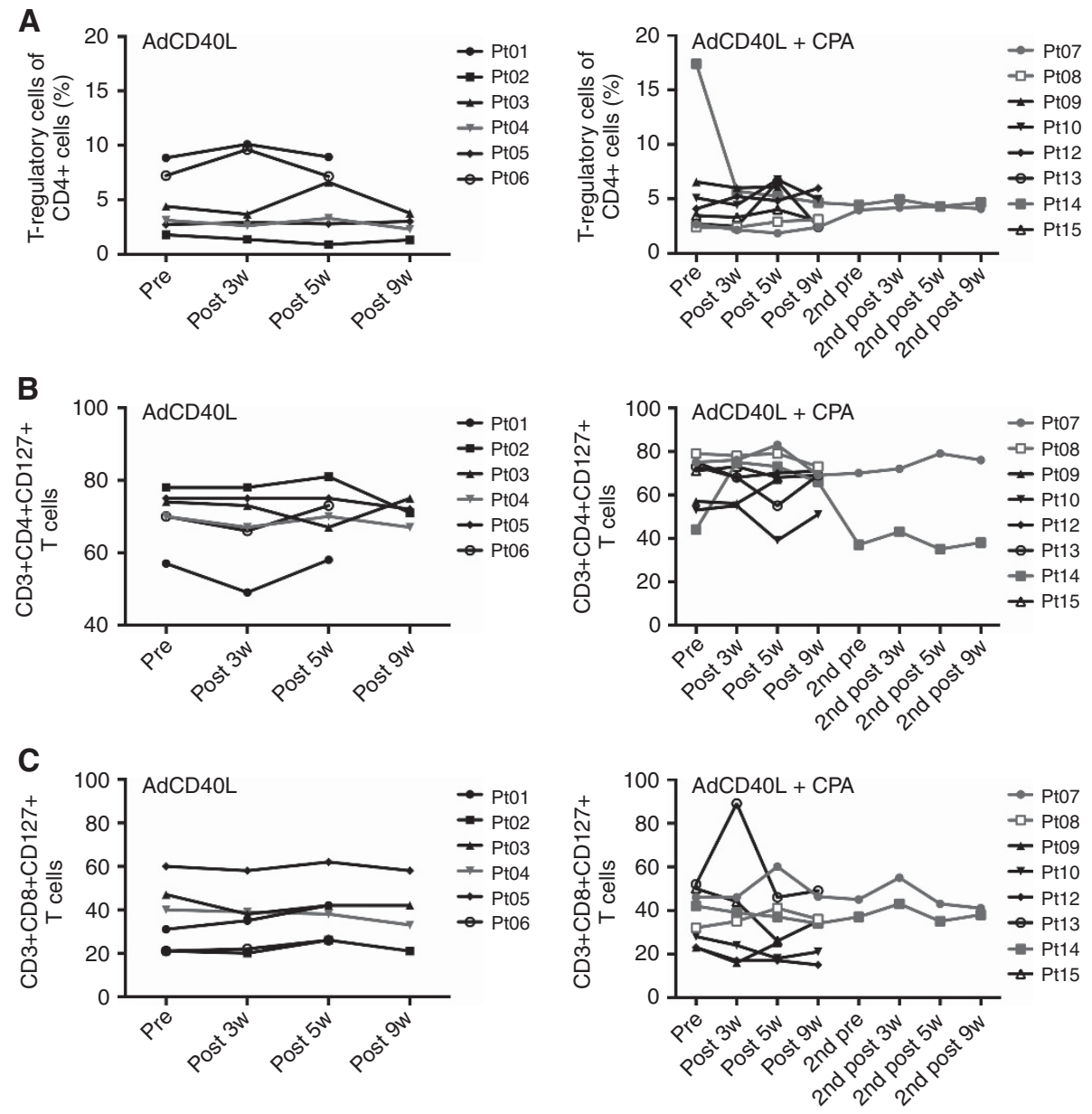

Figure 3. Flow cytometry analysis of PBMCs. Flow cytometry analysis of PBMCs from malignant melanoma patients with advanced disease treated with AdCD40L with (patients \#7 to \#15) or without (patients \#1 to \#6) low dose cyclophosphamide. Time points included for all patients before treatment (Pre) and weeks 3, 5 and $9(3 \mathrm{w}, 5 \mathrm{w}, 9 \mathrm{w})$ post treatment initiation, and for patients \#7 and \#14, the same time points post a second cycle of treatments were tested. Patients \#04,\#7, \#8 and \#14 had the best overall survival and are demonstrated in red in the figure. (A) T-regulatory cells were evaluated as CD3 + CD4 + FoxP3 + CD127 - cells of total CD3 + CD4 + T cells. (B) Activated CD4 T cells were evaluated as CD3 + CD4 + FoxP3-CD127 + cells of total CD3 + CD4 + T cells. (C) Activated CD8 T cells were evaluated as CD3 + CD4 + CD127 + cells of total $\mathrm{CD} 3+\mathrm{CD} 8+\mathrm{T}$ cells.

detected in all the patients at different levels. However, there was no significant difference between pre and post treatment and the expression level did not correlate to OS (Figure 4B). Nevertheless, a high level of CD40 was correlated to a high level of caspase 8 (Figure $4 \mathrm{C}, P=0.0261$ ). CD40 expression was confirmed by immunohistochemistry on tumour cells where high $v s$ low staining were grouped and compared against OS (Figure 4D).

Decreasing levels of IL8 post treatment correlates to overall survival. Increased serum IFN $\gamma$ levels were not detected in any of the patients. Nevertheless, IFN $\gamma$-induced protein 10 (IP10; CXCL10) and Monokine induced by IFN $\gamma$ (MIG; CXCL9) were present (Figure 4E). For the patients experiencing the longest OS, IP10 was highest in the plasma of patient \#4 but that was not reflected in the tumour biopsy, and vice versa for patient \#8. Patient \#7 and \#14 had low but increasing levels in the plasma while decreasing in their tumours despite an initial modest increase in patient \#7. A similar pattern was seen for CXCL9. TGF $\beta$ is an immunosuppressive molecule increased in cancer patients. TGF $\beta$ was detected in all patients and the longest living patients had lower levels than the other patients with the exception of patient \#8 that had initially high levels that were decreased during therapy. While patient \#4 had no detectable IL8, both patient \#7 and \#14 had high levels in the tumour biopsies before treatment but IL8 was decreased substantially during treatment. The fold decrease of IL8 pre $v s$ post therapy was correlated to longer OS $(P=0.0094$, Spearman's, $r=-0.6791$, Supplementary Figure 1).

\section{DISCUSSION}

Malignant melanoma remains one of the most challenging malignancies to treat but it has shown sensitivity for immunotherapy (Lutzky, 2010). The most common adverse events connected to AdCD40L were transient fever, pain at the injection site and transient increase of liver enzymes. A few patients experienced mild nausea after the administration of cyclophosphamide conditioning. One patient reacted with fever, while receiving a blood transfusion because of anaemia of chronic disease just after the third intratumoral injection with the investigational drug. The same patient developed vitiligo-like lesions that were observed 3 weeks after the fourth intratumoral injection. This was the only reaction in the study considered to be of autoimmune nature. We have previously treated patients with bladder cancer AdCD40L (Malmstrom et al, 2010). These patients did not experience adverse events other than transient fever similar to the current melanoma 
A

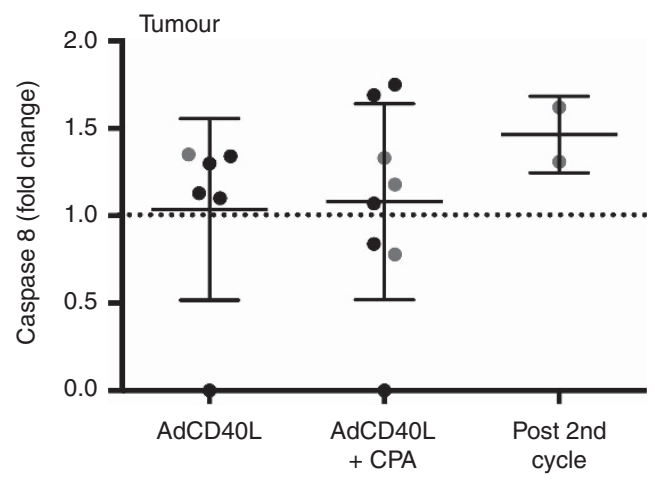

B

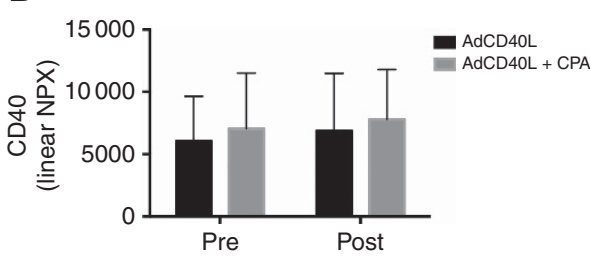

C

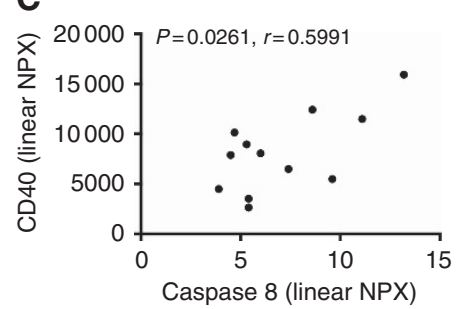

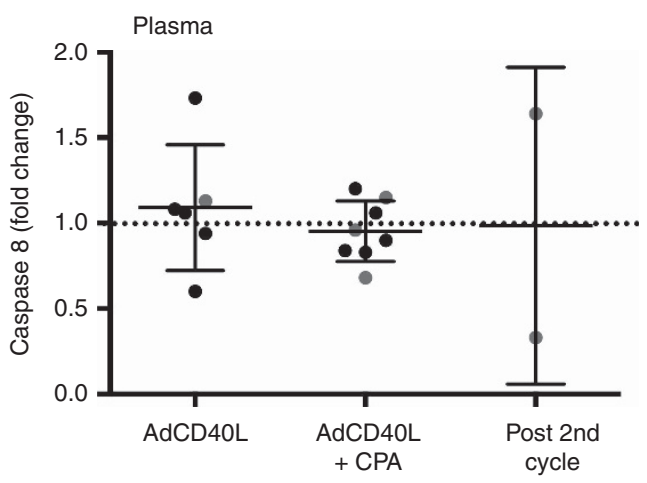

D

E

Plasma

Tumour

Plasma

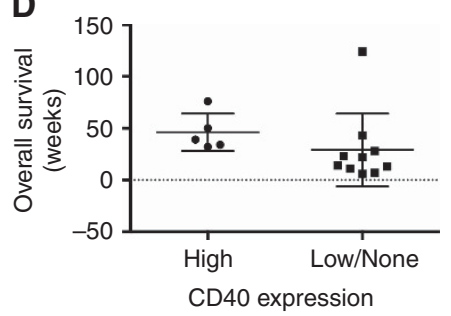

IP10
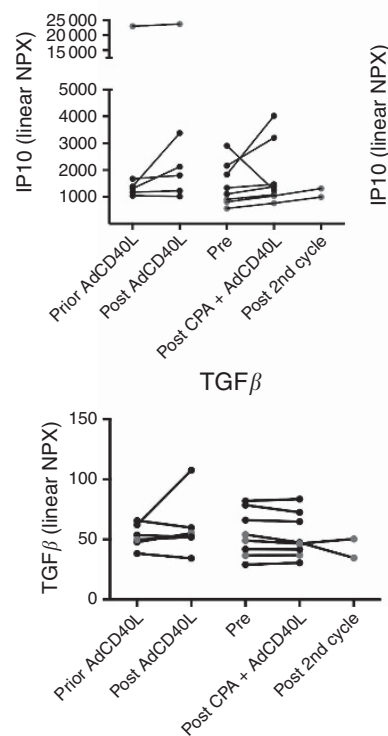

IP10

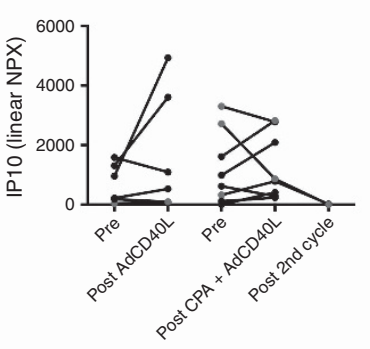

TGF $\beta$

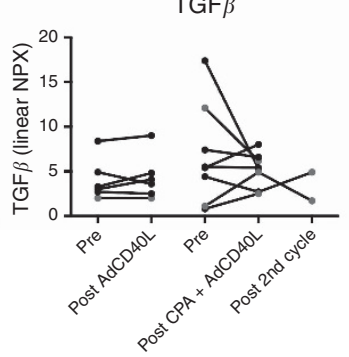

CXCL9
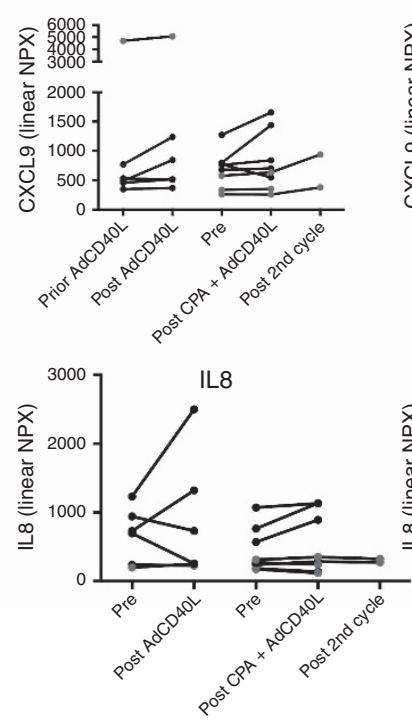

Tumour

CXCL9
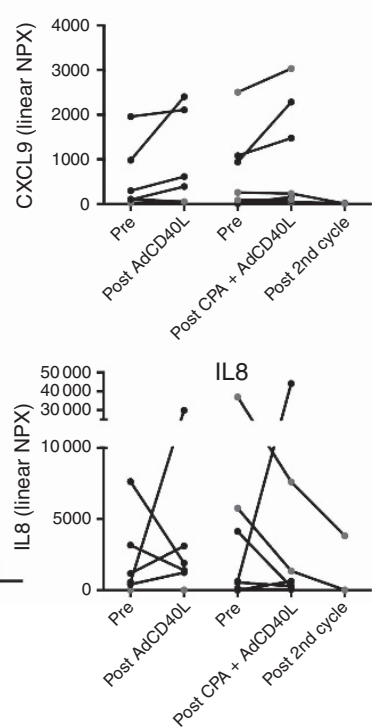

Figure 4. Analysis of plasma and tumour lysates. Plasma and tumour lysates from malignant melanoma patients with advanced disease treated with AdCD40L with $(n=9)$ or without $(n=6)$ low-dose cyclophosphamide were analysed. The ProSeek Inflammation assay was used to determine the levels of caspase 8 (A), CD40 (B), IP10, CXCL9, TGFb and IL8 (E). Correlations between CD40 and caspase 8 levels were assessed (C). CD40 expression was also determined by immunohistochemistry and plotted against overall survival (D). Patients \#4, \#7, \#8 and \#14 had the best overall survival and are demonstrated in red in the figure. Statistical significances were determined by Spearman's correlation test, Student's t-test with Welsh correction (unpaired) or Wilcoxon (paired).

study. Antibodies to the virus vector were increased post treatment but neither the levels prior or post therapy correlated with OS.

AdCD40L therapy reinforced by low-dose cyclophosphamide did not reduce tumour load according to RECIST criteria in any of the patients. Nevertheless, it is common that survival advantages from immunotherapy are not accompanied with ORs (Hodi et al, 2010). In several patients we observed lower metabolic tumour activity at PET/CT after treatment. In seven patients, the SUVmax declined in the injected lesion, two of whom showed a decreased SUVmax in all the metastases while in four of them the SUVmax decreased in some of the distant lesions. These observations indicate that local therapy could induce systemic effects but that the local anti-tumour effect was better. Altogether, we demonstrate at least five patients who experienced clinical benefits of the treatment, Two patients that received AdCD40L only: patient \#2 showed decreased FDG uptake in a distant metastasis and patient \#4 had SD after $2 \frac{1}{2}$ years. Three patients that were conditioned with cyclophosphamide before AdCD40L: patients \#7 and \#8 with clear overall decreased metastatic metabolic activity and patient \#14 who had significant clinical benefit. A second round of therapy was accompanied with improved general condition in one out of our two re-treated patients demonstrating that re-treatment can be 
of value. Furthermore, the 6-month survival was significantly better in the cyclophosphamide group as compared with the AdCD40L-alone group. In addition, the median survival was almost doubled in conditioned patients. Finally, the frequency of adverse events connected to the virus such as flu-like symptoms and pyrexia was increased in the cyclophosphamide group, which also indicates that the likelihood for the virus to induce immune activation was higher in patients conditioned with cyclophosphamide. Nevertheless, these differences in survival have to be interpreted with caution owing to the low number of patients.

In this trial, 15 patients were enrolled, eight with ocular melanoma and five with skin melanoma. Thus, uncommon subgroups of melanoma were over-represented, probably because there are fewer established treatment options for these patients. Owing to the small sample size, it is unclear whether subgroups of melanoma patients respond differently to the treatment but we did not register a different response pattern in patients with ocular melanoma $v s$ other melanomas.

In a previous study, in bladder cancer patients receiving intravesical instillations of AdCD40L, the effector marker IFN $\gamma$ was increased in bladder biopsies post treatment (Malmstrom et al, 2010). In the present study, however, all the patients were heavily pretreated, resulting in a poor immunological capacity. IFN $\gamma$ was below the detection limit, however, IP10 and CXCL9 were detected in both plasma and in tumour biopsies. Interestingly, the long-time survivor patient \#4 had exceptionally high levels of these two proteins in the plasma but not in the tumour. The levels of Tregs and effector $\mathrm{T}$ cells remained stable in this patient. In patients conditioned with cyclophosphamide, circulating Tregs were not significantly reduced, but there was a great variation. Overall, the patients experiencing the longest survival had low Treg levels and the highest levels of phenotypic CD4 and CD8 effector T cells. In addition to the immunostimulatory effect of CD40L, it can induce a signalling cascade leading to apoptosis of CD40 + tumour cells (Loskog and Totterman, 2007). As melanoma commonly expresses CD40, it is a suitable target for CD40L-mediated apoptosis (van den Oord et al, 1996; Tong and Stone, 2003). CD40 could be detected in all the biopsies but at varying levels. Interestingly, a high level of CD40 correlated to a high level of caspase 8 post treatment. Furthermore, decreasing intratumoral levels post treatment of the angiogenic and metastasis promoting cytokine IL8 (Ugurel et al, 2001) correlated significantly to OS.

In summary, this is the first phase I/IIa study evaluating AdCD40L therapy in metastasised cancer patients. In comparison with adoptive T-cell therapy previously tested in our clinic (Bernatchez et al, 2012), treatment with intratumoral AdCD40L was shown to be less resource demanding and less toxic. At least five patients showed clinical benefit of the treatment. Local as well as distant decreased uptakes were seen by PET/CT in seven patients and the best morphological response (RECIST) was SD in 6 of the 15 patients. The results are encouraging and further studies are warranted with possible improvements of the treatment schedule with continuous injections.

\section{ACKNOWLEDGEMENTS}

We are grateful to all patients that participated in this study. The authors thank study nurse Agneta Lindahl for her patient coordination and care as well as Gabriella Paul Wetterberg at Uppsala University for the sample preparation and experimental analyses and Olink Bioscience for the support regarding multiplex analyses. The studies were funded by grants to Professor A Loskog (The Swedish Cancer Society), Professor TH Totterman (The Swedish Cancer Society, Medical Faculty at Uppsala University and
Swedish State Support for Clinical Research (ALF)) and associate Professor Dr G Ullenhag (The research foundation Stiftelsen Onkologiska Kliniken i Uppsala Forskningsfond and Lion's Cancer Fund at Uppsala University Hospital).

\section{CONFLICT OF INTEREST}

Dr Loskog is the $\mathrm{CEO}$ and board member of Lokon Pharma $\mathrm{AB}$, the chairman of Vivolux $\mathrm{AB}$ and RePos Pharma $\mathrm{AB}$, a scientific advisor at NEXTTOBE $\mathrm{AB}$ and has a royalty agreement with Alligator Bioscience AB and Lokon Pharma AB. Dr Tötterman and Dr Mangsbo have a royalty agreement with Alligator Bioscience $\mathrm{AB}$ and are board members and shareholders in Immuneed $\mathrm{AB}$. Dr Mangsbo was $\mathrm{CEO}$ of Immuneed $\mathrm{AB}$ and is employed by AstraZeneca AB. Dr Tötterman is an advisor of Alligator Bioscience AB. Dr Ullenhag was a Medical Advisor to Lokon Pharma AB. The remaining authors declare no conflict of interest.

\section{REFERENCES}

Bernatchez C, Radvanyi LG, Hwu P (2012) Advances in the treatment of metastatic melanoma: adoptive T-cell therapy. Semin Oncol 39: 215-226.

Gehan EA (1961) The determinatio of the number of patients required in a preliminary and a follow-up trial of a new chemotherapeutic agent. J Chronic Dis 13: 346-353.

He TC, Zhou S, da Costa LT, Yu J, Kinzler KW, Vogelstein B (1998) A simplified system for generating recombinant adenoviruses. Proc Natl Acad Sci USA 95: 2509-2514.

Hodi FS, O’Day SJ, McDermott DF, Weber RW, Sosman JA, Haanen JB, Gonzalez R, Robert C, Schadendorf D, Hassel JC, Akerley W, van den Eertwegh AJ, Lutzky J, Lorigan P, Vaubel JM, Linette GP, Hogg D, Ottensmeier CH, Lebbe C, Peschel C, Quirt I, Clark JI, Wolchok JD, Weber JS, Tian J, Yellin MJ, Nichol GM, Hoos A, Urba WJ (2010) Improved survival with ipilimumab in patients with metastatic melanoma. $N$ Engl J Med 363: 711-723.

Hwang JP, Lim I, Na, Cho EH, Kim BI, Choi CW, Lim SM (2014) Prognostic value of SUVmax measured by fluorine-18 fluorodeoxyglucose positron emission tomography with computed tomography in patients with gallbladder cancer. Nucl Med Mol Imaging 48: 114-120.

Loskog A, Totterman TH (2007) CD40L - a multipotent molecule for tumor therapy. Endocr Metab Immune Disord Drug Targets 7: 23-28.

Lutzky J (2010) New therapeutic options in the medical management of advanced melanoma. Semin Cutan Med Surg 29: 249-257.

Malmstrom PU, Loskog AS, Lindqvist CA, Mangsbo SM, Fransson M, Wanders A, Gardmark T, Totterman TH (2010) AdCD40L immunogene therapy for bladder carcinoma-the first phase I/IIa trial. Clin Cancer Res 16: 3279-3287.

Muranski P, Boni A, Wrzesinski C, Citrin DE, Rosenberg SA, Childs R, Restifo NP (2006) Increased intensity lymphodepletion and adoptive immunotherapy-how far can we go? Nat Clin Pract Oncol 3: 668-681.

Sosman JA, Kim KB, Schuchter L, Gonzalez R, Pavlick AC, Weber JS, McArthur GA, Hutson TE, Moschos SJ, Flaherty KT, Hersey P, Kefford R, Lawrence D, Puzanov I, Lewis KD, Amaravadi RK, Chmielowski B, Lawrence HJ, Shyr Y, Ye F, Li J, Nolop KB, Lee RJ, Joe AK, Ribas A (2012) Survival in BRAF V600-mutant advanced melanoma treated with vemurafenib. $N$ Engl J Med 366: 707-714.

Sullivan RJ, Lorusso PM, Flaherty KT (2013) The intersection of immunedirected and molecularly targeted therapy in advanced melanoma: where we have been, are, and will be. Clin Cancer Res 19: 5283-5291.

Tong AW, Stone MJ (2003) Prospects for CD40-directed experimental therapy of human cancer. Cancer Gene Ther 10: 1-13.

Ugurel S, Rappl G, Tilgen W, Reinhold U (2001) Increased serum concentration of angiogenic factors in malignant melanoma patients correlates with tumor progression and survival. J Clin Oncol 19: $577-583$.

van den Oord JJ, Maes A, Stas M, Nuyts J, Battocchio S, Kasran A, Garmyn M, De Wever I, De Wolf-Peeters C (1996) CD40 is a prognostic marker in primary cutaneous malignant melanoma. Am J Pathol 149: 1953-1961. 
Weber J (2007) Review: anti-CTLA-4 antibody ipilimumab: case studies of clinical response and immune-related adverse events. Oncologist 12: 864-872.

Westberg S, Sadeghi A, Svensson E, Segall T, Dimopoulou M, Korsgren O, Hemminki A, Loskog AS, Totterman TH, von Euler H (2013) Treatment efficacy and immune stimulation by AdCD40L gene therapy of spontaneous canine malignant melanoma. J Immunother 36: 350-358.

Young H, Baum R, Cremerius U, Herholz K, Hoekstra O, Lammertsma AA, Pruim J, Price P (1999) Measurement of clinical and subclinical tumour response using [18F]-fluorodeoxyglucose and positron emission tomography: review and 1999 EORTC recommendations. European Organization for Research and Treatment of Cancer (EORTC) PET Study Group. Eur J Cancer 35: 1773-1782.

This work is published under the standard license to publish agreement. After 12 months the work will become freely available and the license terms will switch to a Creative Commons AttributionNonCommercial-Share Alike 4.0 Unported License.

Supplementary Information accompanies this paper on British Journal of Cancer website (http://www.nature.com/bjc) 